\title{
Germanica
}

\section{Zwischen Sinn(en)fülle und Widerstand - Bienen und Wespen als poetologische Symbole in der Lyrik Jan Wagners und Thomas Klings}

Antje Schmidt : Entre profusion sensuelle et résistance. Les abeilles et les guêpes comme symboles poétologiques dans la poésie de Jan Wagner et Thomas Kling Antje Schmidt: Between Sensual Delight and Resistance - Bees and Wasps as Poetological Symbols in Jan Wagner's and Thomas Kling's Poetry

\section{Antje Schmidt}

\section{OpenEdition}

Journals

Édition électronique

URL : http://journals.openedition.org/germanica/6565

DOI : 10.4000/germanica.6565

ISSN : 2107-0784

Éditeur

Université de Lille

Édition imprimée

Date de publication : 26 juin 2019

Pagination : $57-72$

ISBN : 978-2-913857-42-1

ISSN : 0984-2632

\section{Référence électronique}

Antje Schmidt, „Zwischen Sinn(en)fülle und Widerstand - Bienen und Wespen als poetologische Symbole in der Lyrik Jan Wagners und Thomas Klings", Germanica [Online], 64 | 2e trimestre 2019 Online erschienen am: 01 Januar 2021, abgerufen am 23 Februar 2021. URL: http://

journals.openedition.org/germanica/6565 ; DOI: https://doi.org/10.4000/germanica.6565 


\title{
Zwischen Sinn(en)fülle und Widerstand - \\ Bienen und Wespen als poetologische Symbole in der Lyrik Jan Wagners und Thomas Klings
}

\author{
Antje Schmidt \\ Universität Hamburg
}

Die Faszination für das fremdartige Potenzial von Insekten, im Alltag häufig unbeachtet, bietet in der zeitgenössischen deutschsprachigen Lyrik seit einiger Zeit Anlass für literarische Selbstreflexionen. So gilt etwa Durs Grünbein das fortwährende Gestaltwandeln als unverwechselbares Kennzeichen des Typus eines „neue[n] Künstler[s]“1 der 1990er Jahre. Das Schaffen dieses Typus sei nach dem Zusammenbruch des Sowjetregimes in einem Zeitalter des Transits zu verorten, sodass die einzige Beständigkeit seines ästhetischen Schaffens in Prozessen des steten Wandels besteht. Der Transitkünstler, der sich den neuen chronikalischen Verhältnissen des postsowjetischen Zeitalters anzupassen habe, habe daher

1. - Durs Grünbein, „Transit Berlin“, in: Ders., Galilei vermißt Dantes Hölle und bleibt an den Maßen hängen. Aufsätze 1989-1995, Frankfurt a.M., Suhrkamp, 1996, S. 136-143, hier: S. 141. 
kein Programm mehr, sondern nur noch Nerven und einen feinen Spürsinn für Koordinaten. [...] Luftsprünge ins Immaterielle sind seine liebsten Züge in einem Spiel, das seine Regeln immer wieder aufgibt und aufs neue prägt. Stil ist allenfalls noch ironisch spielerische Tarnung oder Mimikry, insektenhafter Bewegungsablauf im Zwielicht von Gewächshausnachmittagen ${ }^{2}$.

Während für Durs Grünbein insektenhafte Beweglichkeit, die dichterische Mimikry, im Vordergrund seines poetologischen Programms steht, betont Thomas Kling die mehrdimensionalen Wahrnehmungsqualitäten von Insekten als ästhetisches Vorbild für seine Lyrikproduktion:

Wie zoomt man auf den Alltag? In der Lyrik der 80er Jahre hat sich gezeigt, daß sich ein mehrdimensionaler Blick, ein Facettenblick wie bei Insekten, besser bewährt, eine inhomogene Gesellschaft und Dinge des Alltags aus überraschenden Blickwinkeln zu schildern. Und dabei ist Sprache gefragt. Streng durchgearbeitete Sprache, die auch der Schönheit der Sprache etwas zutraut ${ }^{3}$.

An sich selbst und ihre Dichterkolleg*innen formulieren zwei der einflussreichsten Lyriker des ausgehenden 20. Jahrhunderts also den poetologischen Anspruch, insektenhaft zu sein, sich der steten lyrischen „Suchbewegung“4 zu verschreiben, Mehrdimensionalität und Multisubjektivität ins Gedicht zu holen und sich somit ostentativ von den Ansprüchen subjektiver Innerlichkeit und Ganzheit zu verabschieden, wie sie angeblich in den 1970er Jahren Einzug in die deutschsprachige Lyriklandschaft gehalten hatten ${ }^{5}$. Im Zuge dieser Denkbewegung erhebt Thomas Kling die angriffslustige, stets unberechenbar agierende und zugleich anmutige Wespe zum zentralen poetologischen Symbol seiner hermetischen Dichtung. Auch in Jan Wagners Lyrik des frühen 21. Jahrhunderts tummeln sich Kleinstlebewesen wie Mücken, Fliegen, Schmetterlinge, Wespen, Bienen und Hummeln. Traditionsbewusst inszeniert dieser das Treiben eines ganzen Bienenschwarms als Allegorie für sein poetologisches Programm der Sinnenfreude und des poetischen Widerstandes.

2. — Ebd. Hervorh. A.S.

3. - Thomas Kling, „Thomas Kling im Gespräch mit Heribert Brinkmann, 2001“, in: Ders., Das brennende Archiv, Unveröffentlichte Gedichte, Briefe, Handschriften und Photos aus dem Nachlaß sowie zu Lebzeiten entlegen publizierte Gedichte, Essays und Gespräche, hrsg. v. Norbert Wehr/Ute Langanky, Berlin, Suhrkamp, 2012, S. 15. Hervorh. A.S.

4. - Hermann Korte, Geschichte der deutschen Lyrik. Bd. 6: Von 1945 bis heute, Stuttgart, Reclam, 2012, S. 82.

5. - Vgl. dazu auch Klings Polemik gegen die „Bauchnabelbetrachter, Fußlahme des Denkens“ der 1970er Jahre. Thomas Kling, „Sprachinstallation 2“, in: Ders., Itinerar, Frankfurt a. M., Suhrkamp, 1997, S. 15-26, hier: S. 21. 
Es ist ausgehend von diesen Beobachtungen das Ziel des vorliegenden Beitrags, die poetologische Bedeutung der schwarz-gelben Hautflüglerinnen im Werk Thomas Klings und Jan Wagners im Spannungsfeld zwischen Tradition und Innovation zu erkunden ${ }^{6}$. Als poetologische Symbole sind die Bienen und Wespen, die sich in den Werken Klings und Wagners vorfinden lassen, insofern zu verstehen, als sie in den Gedichten und essayistischen Schriften der Autoren zentrale produktions-, werk- und rezeptionsästhetische Überlegungen verkörpern und somit im Dienste einer ,individuelle[n] Standortbestimmung des Künstlers" ${ }^{\text {"7 }}$ stehen.

\section{Honigsüßßer Gesang und der Stachel der Inspiration - Bienen und ihre Produkte als poetologische Symbole in der literarischen Tradition}

Der poetische Vergleich zwischen der Tätigkeit der summenden, süßen Honig produzierenden Bienen und den inspirierten, traditionsbewussten Poet*innen und ihren Werken lässt sich von der Antike über die Renaissance bis ins 21 . Jahrhundert verfolgen ${ }^{8}$, wobei die Biene und ihre Produkte in poetologischer Absicht zumeist semantisch mit Attributen wie Harmonie, Schönheit, Göttlichkeit und Fülle verbunden werden ${ }^{9}$. In der Dichtung der griechisch-römischen Antike etwa fungiert der Honig, von dem man annahm, er falle, ,wie eine Art Tau“10 vom Himmel und werde im Anschluss von den fleißigen Bienen bloß noch eingesammelt, als beliebter Vergleichsgegenstand für die „Süße

6. - Lohnenswert wäre diese Unternehmung sicherlich auch in Bezug auf andere zeitgenössische Autor*innen wie etwa Monika Rinck, in deren Honigprotokolle das Gedicht „Honig“ zu finden ist, in dem es in Anspielung auf Platons Ion heißt „Das Honigprotokoll des Bienenflugs / über die von ihnen (den Bienen) gerade noch erreichbaren Blüten. / [...] Nicht bei vernünftigem Bewusstsein / dichten sich diese herrlichen Lieder. Es sammeln die einen für die andern, / und keiner tut etwas für sich ganz alleine.“ Monika Rinck, „Honig“, in: Dies., Honigprotokolle. Sieben Skizzen zu Gedichten, welche sehr gut sind, Berlin, kookbooks, 2014, S. 76.

7. - Olaf Hildebrand, „Einleitung“, in: Ders. (Hrsg.), Poetologische Gedichte von Klopstock bis Grünbein. Gedichte und Interpretationen, Köln, Weimar, Wien, Böhlau, 2003, S. 1-15, hier: S. 6.

8. - Vgl. Günter Butzer/Joachim Jacob, „Biene“, in: Dies. (Hrsg.), Metzler Lexikon literarischer Symbole, Stuttgart, Weimar, Metzler, 2012, S. 44-46, hier: S. 45.

9. - Vgl. Johann Philipp Glock, Die Symbolik der Bienen und ihrer Produkte: In Sage, Dichtung, Kultus, Kunst und Bräuchen der Völker. Eine kulturgeschichtliche Schilderung des Bienenvolkes auf ästhetischer Grundlage, 2. Ausg., Heidelberg, Groos, 1897, S. $200 \mathrm{f}$.

10. - Jan Hendrik Waszink, Biene und Honig als Symbol des Dichters und der Dichtung in der griechisch-römischen Antike, Opladen, Westdeutscher Verlag, 1974, S. $6 \mathrm{f}$. 
und Wahrhaftigkeit des Gesanges"11 der göttlich inspirierten Dichter, welche nicht unmittelbar erkennbare Wahrheiten rhetorisch gewandt und auf prophetische Weise verkündeten ${ }^{12}$.

Zum Symbol der Person des inspirierten Dichters werden die Honigbienen in der griechisch-römischen Antike durch eine poetische Akzentverschiebung vom Werk auf das dichtende Subjekt ${ }^{13}$. In Platons Ion werden die göttlich inspirierten Dichter wirkmächtig mit der Biene verglichen, die im Zustand der Verzückung ,,ihre Lieder ernten und uns bringen, wie die Bienen den Honig“, dabei aus den „honigströmenden Quellen der Musen“ schöpften und „der Musik [...] und Rhythmus [verfallen]" seien ${ }^{14}$. Waszink betont zudem, dass mit der Akzentverschiebung auf das Dichtersubjekt „die Abwechslung, die Buntheit, die Farbenfreudigkeit" 15 als zusätzliches Merkmal der durch Bienen symbolisierten Dichtkunst erstmalig in der Antike in Erscheinung trete - denn wie die Biene von Blüte zu Blüte fliegt, kann auch der Dichter aus der Fülle der Wirklichkeit schöpfen.

Dieselbe Allegorie bemüht Seneca in seinem berühmten Bienengleichnis ${ }^{16}$ für den fleißigen, nachahmenden Dichter, der den Nektar seiner literarischen Vorbilder verdaut und diese anschließend im Sinne der imitatio produktiv in den süßen Honig der Dichtung transformiert ${ }^{17}$. Ausgiebig rezipiert wurde das Bienengleichnis in der Renaissance $^{18}$. Bei Petrarca tritt schließlich der dichterische Anspruch hinzu, die gesammelten und assimilierten Stimmen der fremden Dichter $\mathrm{zu}$ dissimilieren, um etwas Neues aus dem vorliegenden Material zu erschaffen und einen eigenen, unverwechselbaren Stil zu entfalten ${ }^{19}$ :

Dies aber bekräftige ich: Daß es eine elegantere Kunstfertigkeit ist, wenn wir, als Nachahmer der Bienen, mit unseren Worten auch beliebig viele Sätze fremder Menschen vortragen. Allerdings sollen wir nicht den Stil von diesem oder jenem haben, sondern den einen unseren, aus mehreren zusammengeschmolzenen ${ }^{20}$.

11. — Ebd. Hervorh. i.O.

12. - Vgl. ebd., S. 7-12.

13. - Vgl. J. H. Waszink, Biene und Honig als Symbol..., a.a.O., S. 14.

14. - Platon, Ion 534a u. 534b. Verwendete Ausgabe: Platon, Ion oder Über die Ilias, übers. u. komm. v. Ernst Heisch. Göttingen, Vandenhoeck \& Ruprecht, 2017, S. 15.

15. - J. H. Waszink, Biene und Honig als Symbol..., a.a.O., S. 14.

16. - Vgl. Seneca, Epistulae morales 84.

17. - Vgl. G. Butzer/J. Jacob, „Biene“, a.a.O., S. 45.

18. - Vgl. Klaus Irle, Der Ruhm der Bienen. Das Nachahmungsprinzip der italienischen Malerei von Raffael bis Rubens, Münster u.a., Waxmann, 1997, S. 7.

19. - Vgl. Aura Maria Heydenreich, Wachstafel und Weltformel, Erinnerungspoetik und Wissenschaftskritik in Günter Eichs ,,Maulwürfen“, Göttingen, Vandenhoeck \& Ruprecht, 2007, S. 94.

20. - Petrarca, La Familiari, I, 7. Übersetzung zit. nach A. M. Heydenreich, Wachstafel und Weltformel, a.a.O., S. 94. 
Das Verdienst der Bienen bestehe darin, dass sie „das Vorgefundene in etwas Anderes und Besseres verwandeln" 21 .

Doch neben der dominanten Traditionslinie ist auch der gefährliche Stachel der Biene im Vergleich zwischen Bienen und Dichtkunst traditionell von Bedeutung, etwa bei Valéry, in dessen Gedicht L'abeille „die rasche Pein eines [Bienen]stichs verlangt wird, die auch als Bitte um augenblickshafte [...] poet[ische] Inspiration zu verstehen ist" ${ }^{\text {"22 }}$.

\section{Bienen als magisches Material - Jan Wagners „selbstporträt mit bienenschwarm“"}

Jan Wagner, dessen dichterisches Werk insbesondere im Zuge der Verleihung des Preises der Leipziger Buchmesse auch von einem breiteren Publikum rezipiert wurde, gilt einigen seiner Kritiker*innen als Traditionalist ${ }^{23}$, der zum Konventionellen neige. Dass er für seine poetologische Selbstverortung gleichermaßen traditionsbewusst die Biene als sein dichterisches Wappentier erwählt, nährt zwar diesen Verdacht, doch dieser erweist sich bei genauerem Hinsehen als unbegründet. Denn der besondere Reiz der Lyrik Jan Wagners liegt gerade in der spielerischen Neuinszenierung von scheinbar Altbekanntem. Auch in seinem zentralen poetologischen Gedicht selbstporträt mit bienenschwarm ${ }^{24}$, das zugleich titelgebend für seinen 2016 erschienenen lyrischen Auswahlband war, verfährt Jan Wagner in Bezug auf die Tradition der Bienensymbolik in spielerisch-aneignender Weise.

\section{selbstporträt mit bienenschwarm}

bis eben nichts als eine feine linie um kinn und lippen, jetzt ein ganzer bart, der wächst und wimmelt, bis ich magdalena zu gleichen scheine, ganz und gar behaart

von bienen bin. wie es von allen seiten heranstürmt, wie man langsam, gramm um gramm an dasein zunimmt, an gewicht und weite, das regungslose zentrum vom gesang ...

ich ähnele mit meinen ausgestreckten armen einem ritter, dem die knappen

21. — Ebd. Vgl. auch G. Butzer/J. Jacob, „Biene“, a.a.O., S. 45.

22. - G. Butzer/J. Jacob, „Biene“, a.a.O., S. 44.

23. - Vgl. dazu Michael Braun, „Das regungslose Zentrum von Gesang. Zwei Fußnoten zur Dichtkunst Jan Wagners", in: Frieder von Ammon (Hrsg.), Text + Kritik 210: Jan Wagner, München, Richard Boorberg, 2016, S. 46-50, hier: S. 46 ff.

24. - Jan Wagner, „selbstporträt mit bienenschwarm“, in: Ders., Regentonnenvariationen, Berlin, Hanser, 2014, S. 97. 
in seine rüstung helfen, stück um stück, erst helm, dann harnisch, arme, beine, nacken,

bis er sich kaum noch rühren kann, nicht läuft, nur schimmernd dasteht, nur mit ein paar winden hinter dem glanz, ein bißchen alter luft, und wirklich sichtbar erst mit dem verschwinden.

Jan Wagner wählt für seine lyrische Standortbestimmung das Bild eines singenden Bienenschwarmes, der sich von Kopf bis Fuß um das artikulierte Ich des Gedichtes formiert, es nachzeichnet und in eine Rüstung hüllt, bis es in der vierten Strophe hinter Glanz und Luft verschwindet. Dabei offenbart bereits der erste - sich gewissermaßen selbst performativ bestätigende - Vers ,bis eben nichts als eine feine linie“ (V. 1) die Selbstreferenzialität des Gedichtes; die Bienen werden somit lesbar als Zeichen für das physische Sprachmaterial des Gedichtes, für Schrift und Klang. Ebenso wie der fiktive Bienenschwarm das Ich im Gedicht Stück für Stück nachzeichnet, ergibt die Kombination aus der Buchstabenmaterie, den Sätzen und Worten für die realen Leser*innen im Lektüreakt sukzessive die imaginäre Evokation des in den singenden Bienenschwarm gehüllten Ichs.

Wagner, der grundsätzlich skeptisch gegenüber Formen des „populären Sentimentalitätenhandel[s]“"25 in der Lyrik ist, spielt hierbei mit der bis heute wirksamen Gattungstradition der Hegel'schen Erlebnislyrik, die dem Ich im Gedicht „eine engere Verbindung zur Subjektivität und besonders auch zur Persönlichkeit des Autors" 26 und somit der gesamten Gattung einen gewissen Hang zur Innerlichkeit und Sentimentalität unterstellt ${ }^{27}$. Doch das artikulierte Ich im selbstporträt mit bienenschwarm ist gerade nicht mit dem Autor Jan Wagner zu verwechseln ${ }^{28}$, sondern es entsteht in der vorgefundenen Form letztlich erst aus und mit Hilfe der Bienenschrift und ihrem ,gesang“ (V. 8).

Denn gewissermaßen transformieren die Bienen in ihrem Nachahmungsvorgang das Ich, bis sie es ,zur Kenntlichkeit entstellt ${ }^{\star}$ haben, bis es ,wirklich sichtbar erst mit dem verschwinden“ (V. 16) wird. Damit wird im Gedicht ein Vorgang performativ inszeniert, der stark an Petrarcas Ambition aus dem Bienengleichnis erinnert, das

25. - Jan Wagner, „Ins Unbekannte. Über neue Gedichte“, in: Ders., Die Sandale des Propheten. Beiläufige Prosa, Berlin, Berlin Verlag, 2011, S. 22-31, hier: S. 29.

26. - Dieter Burdorf, Einführung in die Gedichtanalyse, 3. Aufl., Stuttgart, Metzler, 2015, S. 183.

27. - Diese Konnotationen trägt auch der Begriff des „lyrischen Ichs“, weshalb er in diesem Beitrag vermieden wird.

28. - Vgl. dazu auch Mirjam Springer, „Selfie mit Bienen. Jan Wagners Spiegelblicke“, in: F. v. Ammon (Hrsg.), Text + Kritik 210: Jan Wagner, a.a.O., 2016, S. 51-63. 
Vorgefundene mit den Mitteln der Dichtung in etwas Anderes - und Besseres $-\mathrm{zu}$ verwandeln ${ }^{29}$ : Wagners Sprachmaterial transformiert einen kleinen Ausschnitt der Wirklichkeit in Poesie, indem er ihn auf unerwartete Weise sprachlich repräsentiert. Diese Poetik des neuen Sehens expliziert Wagner in seinem poetologischen Essay Die Sandale des Propheten. Dort schreibt er, das Gedicht

nimmt sich das Recht, die Dinge so zu denken und zu sehen, wie sie nie zuvor bedacht und gesehen worden sind, und lädt den Leser, seinen Partner, dazu ein, es ihm gleichzutun. Es verhilft dem geflissentlich Übersehenen zu seiner verdienten Aufmerksamkeit und läßt das nur scheinbar Banale leuchten ${ }^{30}$.

„[S]chimmernd“ (V. 14) und voll ,glanz“ (V. 15) wird das nur scheinbar Banale dabei durch die Möglichkeiten der Sprache, wobei produktionsästhetisch „Sprachbetrachtung und Abschweifung“31 gleichermaßen zum gelungenen Gedicht beitragen. Dieses poetische Verfahren verhilft dem bedichteten Gegenstand schließlich dazu, dass es ,langsam, gramm um gramm / an dasein zunimmt, an gewicht und weite" (V. 6f.) - die Wirklichkeit als sprachlich überformte Wirklichkeit gewinnt an Bedeutungsreichtum, ebenso wie das Ich im Gedicht durch die Bienen an Fülle gewinnt. Anders als bei Thomas Kling, bei dem, wie zu sehen sein wird, das Sprachmaterial sich als wesentlich widerspenstiger erweist und daher stärker die Aufmerksamkeit der Leser*innen einfordert, überwiegt in Wagners Lyrik die sprachliche Evokationskraft, die zunächst versucht, die „Normalität [...] staunenswert“ und nach Auffassung Wagners auch ,schöner" werden zu lassen ${ }^{32}$.

Heinrich Detering bemerkt daher äußerst treffend, Wagners Schreiben sei eines der ,weit geöffnete[n] Augen" 33 - und der weit geöffneten Ohren, so ließe sich anschließen. Denn das sprachliche Material präsentiert sich in Wagners Selbstporträt als ein klingendes, summendes, singendes: Verse in fünfhebigen Jamben, angelehnt an den schwingenden Vers commun, gewagte Enjambements wie ,ausgestreck- / ten“ (V. 9f.) und sogenannte slant rhymes, also unreine Reime, bestehend aus

29. - Wobei Petrarca hierbei die literarische Tradition und nicht die Gänze der außerliterarischen Wirklichkeit im Sinne hatte.

30. - Jan Wagner, „Die Sandale des Propheten“, in: Ders., Die Sandale des Propheten..., a.a.O., S. 17-21, hier: S. 21. Dass diese Poetik starke Parallelen zu Viktor Šklovskijs Verfremdungstheorie aufweist, hat Detering gezeigt. Vgl. Heinrich Detering, "Qualle und Killer. Eine Einführung in das Schreiben Jan Wagners", in: F. v. Ammon (Hrsg.), Text + Kritik 210: Jan Wagner, a.a.O., S. 7-14, hier: S. 10.

31. - Jan Wagner, „Lob der Unschärfe“, in: Ders., Die Sandale des Propheten..., a.a.O., S. 32-36, hier: S. 36.

32. - Ebd.

33. - H. Detering, „Qualle und Killer“, a.a.O., S. 13. 
Konsonanzen und Assonanzen, die dezent kreuzgereimt sind, wie etwa „knappen“ (V. 10) und „nacken“ (V. 12) ${ }^{34}$, verleihen dem Gedicht seine besondere Rhythmizität und eine fröhlich-spielerische Ausgelassenheit. Damit führt Wagners selbstporträt mit bienenschwarm die dichterische Tradition der Bienen als Symbole für poetische Fülle, Rhythmus und Harmonie spielerisch fort: „Die Sinnen- und Sinnfülle zu beweisen wäre die Aufgabe des Gedichts"35, so formuliert Wagner es selbst und vollbringt sogleich den Beweis in seinem poetologischen Selbstporträt.

Bleibt zuletzt die Frage nach der Bienenrüstung, die den reglosen „ritter“ (V. 10) wappnet - doch wogegen eigentlich? Braun liest die Rüstung als ,ein ironisches Bild für den Dichter, der hier eine alte

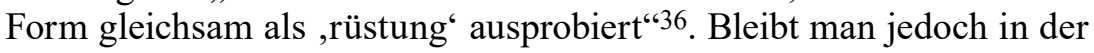
in diesem Beitrag vorgeschlagenen Lesart, die Bienen als selbstreferenzielles Symbol für das dichterische Sprachmaterial zu lesen sowie für seine Funktion, Sinn- und Sinnenfreude hervorzubringen, ist es eine weitere Möglichkeit, das Ich in der glänzenden Bienenschwarmrüstung als eine Allegorie für Wagners Lyrik als das zu lesen, was er das ,trotzige Dennoch"37 nennt. Die Poesie lehnt sich nach Wagner in ihrer Formschönheit und poetischen Nutzlosigkeit widerspenstig gegen das „rein[e] Kosten-Nutzen-Denken“ der heutigen Zeit auf und regt den Lesenden an, an ihrem subversiven Spiel teilzuhaben:

Sie verweigert sich dem Warencharakter, dem Warenstrom, sie ist schlechthin unkonsumierbar und schon dadurch ein politischer Akt, selbst da, wo sie sich nicht vordergründig politisch gibt. Das Gedicht ist auf herrliche Art und Weise vollkommen nutzlos - so nutzlos wie ein Lachen nutzlos ist, ein jähes Glücks- und Hochgefühl, ein absichtsloses Spiel ${ }^{38}$.

Widerstand, aber auch Schutz und Trost verspricht die Bienenschwarmrüstung der Wagner'schen Poesie, da sie „eine lösende, eine erlösende Wirkung haben kann, weil [sie] den Dingen, den Fragwürdigkeiten, eine alle Sinne und unser ästhetisches Empfinden befriedigende und damit letztlich trostreiche Form verleiht"“39. Damit erweist sich der Bienenschwarm als poetologisches Symbol sowohl für trostreiche Fülle und Schönheit der Lyrik Jan Wagners wie auch für ihren Anspruch, stachelbewaffnet ästhetischen Widerstand gegen die Effizienzmechanismen westlicher Konsumkulturen zu leisten.

34. - Vgl. ebd., S. 11.

35. - J. Wagner, „Lob der Unschärfe“, a.a.O., S. 35.

36. - M. Braun, „Zwei Fußnoten zur Dichtkunst Jan Wagners“, a.a.O., S. 49.

37. - J. Wagner, „Die Sandale des Propheten“, a.a.O., S. 20.

38. - Ebd.

39. - Jan Wagner „Der verschlossene Raum“, in: Ders., Der verschlossene Raum. Beiläufige Prosa, Berlin, Carl Hanser, 2017, S. 23-48, hier: S. 44. 
Jan Wagner präsentiert sich also in seinem selbstporträt mit bienenschwarm ebenso formvollendet wie traditionsbewusst als Bienendichter, der an sein Werk den Anspruch der trostreichen Sinn- und Sinnenfülle und der poetischen Widerständigkeit vereint. Die Welt des Gedichtes, so führt es Wagners Selbstporträt vor, ist ein eigener, nahezu magischer Wirklichkeitsraum, geschaffen aus nichts als Buchstaben, Lauten und der Vorstellungskraft des Schreibenden sowie der Lesenden.

\section{Thomas Kling - Übermittlung von „wespenfrequenzen“}

Der Poet Thomas Kling hingegen präsentiert sich in seinen Gedichten und Performances als Gegenentwurf zum von Platon beschworenen, von Harmonie erfüllten Bienendichter. Denn der Sprachinstallateur und „Fährtenleger"40 Kling war, so formuliert er es selbst, eine aggressive und theateraffine „Rampensau“41. $\mathrm{Zu}$ seiner wohldurchdachten, eigensinnigen und stets expressiven Inszenierung ${ }^{42}$ zählte bei seinen zahlreichen Lesungen und Auftritten daher mit Vorliebe sein schwarz-gelb-gestreifter Wespenpullover. Diese Beobachtung und die rasanten Lesungen Klings veranlassten Hubert Winkels dazu, die Wespe als eines von zwei Wappentieren des Dichters zu küren:

Die Wespe ist ein Lieblingstier von Thomas Kling. Wenn Feudalherren der Poesie heute Wappen anfertigen ließen, Kling trüge das der stachelbewehrten Schönen, die Aggressivität und Schnelligkeit vereint ${ }^{43}$.

40. - Marcel Beyer, „Thomas Kling: Herz“, in: T. Kling, Das brennende Archiv..., a.a.O., S. 175-187, hier: S. 175.

41. - Marcel Beyer/Thomas Kling, ,Das Eingemachte, Marcel Beyer und Thomas Kling beim Smalltalk, Auszüge“, in: T. Kling, Das brennende Archiv..., a.a.O., S. 4349, hier: S. 46.

42. - Vgl. Frieder von Ammon/Peer Trilcke, „Einleitung“, in: Dies./Alena Scharfschwert (Hrsg.), Das Gellen der Tinte. Zum Werk Thomas Klings, Göttingen, Vandenhoeck \& Ruprecht, 2012, S. 9-22, hier: S. 10.

43. - Die andere Seite des Wappens, so Winkels, wäre „dem Hirschen reserviert“. Hubert Winkels, „Botschaft aus Blei. Vom Tanzboden zum Schlachtfeld“, in: Ders., Stimmen der Ordnung: Über Thomas Kling, Köln, DuMont, 2005, S. 27-39, hier: S. 28. Leider konnte der kürzlich von Achim Geisenhanslücke zum Thema veröffentlichte Beitrag für diese Untersuchung nicht mehr berücksichtigt werden, da er bereits nach der Endredaktion publiziert wurde. Vgl. Achim Geisenhanslücke, „Der Stachel der Dichtung. Thomas Kling, der europäische Wespendichter", in: Frieder von Ammon/ Rüdiger Zymner (Hrsg.), Gedichte von Thomas Kling. Interpretationen, Paderborn, Mentis, 2019, S. 37-48. 
Dass Kling, ,in seiner Karriere als Wespendichter, das bislang sicher umfang- und facettenreichste Wespenwerk in Versen geschaffen hat ${ }^{\text {“44, }}$ bemerkt auch Tobias Lehmkuhl, der dem aus dem Geiste des Punk geborenen Poeten ${ }^{45}$ mit seiner ,,spitzen Feder ${ }^{646}$ zudem attestiert, stets der Stachel im Fleische des deutschen Literaturbetriebes gewesen zu sein ${ }^{47}$.

Stellt die Wespe offensichtlich ein Element in Thomas Klings künstlerischem Schaffen dar, das eine wesentliche Stellung in seiner performativen Inszenierung von Autorschaft sowie wesentlich in seinen schriftlich fixierten Gedichten innehat, greift Winkels Betonung von Schönheit und Schnelligkeit der Kling'schen Dichtkunst zu kurz, um die diffizile semantische Codierung der schwarz-gelben Hautflüglerin in seinem CEuvre zu erfassen. Denn ihr künstlerischer Einsatz sei gar eine „Sache von emblematischer Ernsthaftigkeit, wie sie eigentlich nur das 16./17. Jahrhundert zu bieten hatte" 48 , so formuliert es Kling in einem Interview mit dem Literaturwissenschaftler Hans Jürgen Balmes. Dabei sei „niemals naturwissenschaftliche Coolness“49 das Motiv für seine vielfältig auftauchenden biologischen Embleme. Diesem Hinweis Klings folgend kann die Wespe - und dies anhand einiger Fährten in Klings Werk zu plausibilisieren sei nachfolgend der Anspruch - unter anderem als Symbol für eine mitunter existenzielle poetologische Dimension des Kling'schen Schreibens gelesen werden, für ein Programm, das den Anspruch einer traditionsbewussten dichterischen Selbstbehauptung und die Dimension des Werdens und Vergehens zusammen denkt.

\section{„eine in wut und puls hinfliegende schrift" - Klings unberechenbare Wespenschrift}

In seinen Gedichten und Essays war Thomas Kling stets bestrebt, verschiedene „Genealogien und Traditionen“ $50 \mathrm{zu}$ konstruieren und somit bisweilen ganz eigene Kanonisierungsprozesse der vermeintlich marginalisierten literaturhistorischen Persönlichkeiten voranzutreiben ${ }^{51}$. Als Teil seiner genealogischen Bestrebungen ist auch die

44. - Tobias Lehmkuhl, „Von Bienen und Wespen. Thomas Klings gelbschwarze Dichtung“, Neue Rundschau 115.4/2004, S. 137-149, hier: S. 138.

45. - Vgl. dazu Enno Stahl, „Die Geburt des Geschmacksverstärkers aus dem Geiste des Punk“, in: F. v. Ammon/P. Trilcke/A. Scharfschwert (Hrsg.), Das Gellen der Tinte..., a.a.O., S. 69-80.

46. - T. Lehmkuhl, „Von Bienen und Wespen“, a.a.O., S. 147.

47. - Vgl. ebd.

48. - Hans Jürgen Balmes, „Lippenlesen, Ohrenbelichtung. Ein Gespräch mit Thomas Kling", in: Text + Kritik 147: Thomas Kling, München, Richard Boorberg, 2000, S. 14-23, hier: S. 18.

49. - Ebd.

50. - F. von Ammon/P. Trilcke, „Einleitung“, a.a.O., S. 16.

51. — Vgl. Peer Trilcke, Historisches Rauschen. Das geschichtslyrische Werk 
in Klings Band fernhandel (1999) aufzufindende ,ahnenstrecke“52 zu verstehen, zu der neben Catull, Giacomo Leopardi und Oswald von Wolkenstein auch Annette von Droste-Hülshoff zählt. Er widmet der Dichterin einen umfänglichen Gedichtzyklus mit dem Titel Spleen. Drostemonolog, der sich aus insgesamt 15 Teilgedichten zusammensetzt, die von ihm jeweils als durchnummerierte Findlinge, Kartographien und Daguerreotypien benannt wurden ${ }^{53}$. In diesem Zyklus geht es, die Gedichttitel legen es bereits nahe, um eine Vermessung des Feldes, um die Arbeit mit und am historischen Material, mit dem der „Memorizer“ 54 Kling seine persönlichen Traditionslinien zu Annette von Droste-Hülshoff stiftet ${ }^{55}$. Mirjam Springer hat in ihrem Beitrag überzeugend gezeigt, inwiefern Kling in diesem Zyklus ein ,poetologisches Spiegelporträt" 56 entwirft, in dem er anhand des vorgefundenen Materials gleichermaßen Fremd- und Selbstbespiegelung betreibt.

Für Klings lyrische Selbstverortung ist insbesondere das Wespengedicht des Zyklus aufschlussreich, in dem er sich einerseits, auf einer konkreten Ebene, mit der ,wüst[en]" und teilweise unlesbaren Handschrift Droste-Hülshoffs auseinandersetzt, in deren Materialität

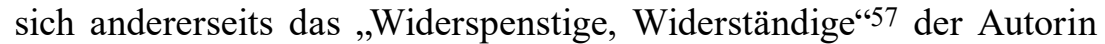
widerspiegelt. In einer poetologischen Lesart kann die im Gedicht evozierte Wespenschrift der Droste zudem als Allegorie auf seine Poetologie der bewegten Schrift ${ }^{58}$ gelesen werden, was wiederum Rückschlüsse auf seine Semantisierung der Wespe als poetologisches Symbol zulässt. Anders als die Biene in der Antike zentrales Symbol für den inspirierten Dichter ist, fungiert die Wespe im nachfolgend analysierten Gedicht primär als werkästhetisches poetologisches Symbol für die wespenhaft bewegte Lyrik Klings. Die Elemente, die dabei zentral erscheinen, seien nachfolgend in der gebotenen Kürze angesprochen.

Thomas Klings, 2012, Online unter: http://hdl.handle.net/11858/00-1735-0000-0006AEDE-3, letzter Zugriff: 7.12.2018, S. 36 f.

52. - Thomas Kling, „Der Schwarzwald 1932“, in: Ders., fernhandel. Gedichte, Köln, DuMont, 1999, S. 77-82, hier: S. 82.

53. - Vgl. T. Kling, fernhandel..., a.a.O., S. 83-99. Die einzige Ausnahme von diesem Kompositionsprinzip bildet das „Westphälische Kehlchen“ zwischen der zweiten und dritten Kartographie. Vgl. ebd., S. 92.

54. - T. Kling, ,Sprachinstallation 2“, a.a.O., S. 16.

55. - Auf die Bezugnahmen auf Droste-Hülshoff kann im Rahmen dieses Beitrages nur am Rande eingegangen werden, sie sind aber hervorragend zusammengefasst in Mirjam Springers Aufsatz. Vgl. Mirjam Springer, „"sounds vom schreibgebirge”. Thomas Klings Zyklus "Spleen. Drostemonolog” (1999)", in: Jochen Grywatsch/ Winfried Woesler (Hrsg.), Droste-Jahrbuch 10/2013/14, S. 205-246.

56. - Ebd., S. 209.

57. - Ebd., S. 229.

58. - Vgl. dazu ebd., S. 228. 


\section{Zweiter Daguerreotyp}

eine in wut und puls hinfliegende schrift:

handschrift, die sich überlagert, blöcke

bildet. buchstabm, eignsinnign buchstabm,

die sich formieren vor ihren augn, auf ab-

gerissenen papieren. blöcke bilden, wort-

kartausen. zitternd hinfliegende, wüst

fallende tinten. handschrift-morganen!

durch die, gestrüpp, mit mühe manchmal nur (...)

drang, andrang von schrift, von handschrift-

morganen: voll abgefeuerter insektencodes;

alles voller fühler, voll aufstampfender wespenfuß.

füße, lippen, wespen, die über bögen gehen, sich

bewegen, rastlos ihre fährten legen: mit wespenfrequenzen

versehene, mit wespenfrequenzen versetzte schrift.

die kleinen eigensinnigen kratzenden buchstabm.

das schneiden und kratzen. aufs sopha gefläzt, aber

lauernd. schriftbereitschaft, mündung, kiel. schon

schriftbereit, in hock-, in kribbelnder lauer-

stellung. in hockstellung auf verkommenem meuble.

es zischen die schwalben ihr durchs arbeitszimmer.

westlage, die wespen, schwalbenkot.

fliegende, wieder und wieder anfliegende, abdrehende,

in hockstellung abreißende, hinreißende schrift ${ }^{59}$.

Nähert man sich dem Verfahren dieses überaus vielschichtigen Gedichtes an, so fällt auf, dass das eigenwillig-aggressive Verhalten der fliegenden Wespen den zentralen Bildspender für die Schriftmetaphern darstellt, was sowohl eine Analogisierung der beiden Phänomene als auch eine Personifizierung der Schrift bewirkt, die somit als wespenhaft attribuierte Akteurin, als eine aggressiv-unruhige Wespenschrift, auftritt.

So wird die Schrift Droste-Hülshoffs etwa im ersten Vers metaphorisch als eine ,in wut und puls hinfliegende schrift" (Hervorh. A.S.) präsentiert, die sich aus ,buchstabm, eignsinnig[en] buchstabm“(V. 3), die ,schneiden und kratzen“ (V. 16) sowie aus ,,zitternd hinfliegende [n], wüst fallende [n] tinten" (V. 6f., Hervorh. A.S.) zusammensetzt. Auch die evozierte Überlagerung der Handschrift (vgl. V. 2) und das „sich formieren“ (V. 4) der Buchstaben lässt an einen Wespenschwarm denken, in dem die einzelnen Insekten sich so eng anordnen, dass sie voneinander ununterscheidbar werden und gemeinsam ,blöcke bilden“ (V. 5). In diesen Versen stehen die Wespen als Bildspender nicht nur für das konkrete, undurchdringliche Schriftbild der Droste, sondern auf einer allegorischen Ebene für eine Undurchdringlichkeit, Wehrhaftigkeit und Widerspenstigkeit des 
Schreibens, wie sie Kling in der Droste'schen Handschrift identifizierend $\mathrm{zu}$ seinem eigenen Schreiben erkennt: „Dichtung wird damit zu einer Praktik der Selbstbehauptung des Gedichts wie des Dichters" 60 , für welche die angriffslustige Wespe allegorisch steht.

Betont werden neben einer aggressiven dichterischen Haltung, die sich im Geschriebenen widerspiegelt, jedoch auch die Qualitäten einer hermetischen Dichtung als ,voll abgefeuerter insektencod[e]“" (V. 10, Hervorh. A.S.), der sich auf rezeptionsästhetischer Ebene dem unmittelbaren Verstehen widersetzt und somit stets zwischen ,Kommunikabilität ${ }^{\star}$ und ,Inkommunikabilität ${ }^{\star}$ steht ${ }^{61}$. Diesen beiden Polen entsprechen im vorliegenden Gedicht einerseits die aufscheinenden „handschrift-morganen“ (V. 7, V. 9f.) und andererseits das schwer zu durchdringende ,gestrüpp“ (V. 8) der Handschrift. Ein Grund für dieses Spannungsfeld, in dem das Kling'sche Schreiben ebenso wie das Droste-Hülshoffs zu verorten ist, ist eine spezifische Bewegtheit des Sprachmaterials. Im Verhalten der unruhigen Wespen, ,die über bögen gehen, sich / bewegen rastlos ihre fährten legen" (V. 12) und somit

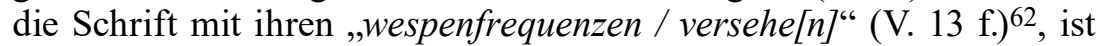
diese ästhetische Bewegung versinnbildlicht, die Trilcke in Anlehnung an Klings Vokabular „Zapping“63 oder „bewegte Struktur"64 nennt. Damit sind insbesondere Verfahren der textuellen Strukturierung gemeint, durch die die syntagmatisch aneinandergefügten Sequenzen teilweise dekontextualisiert und fragmentarisiert sind, so dass für die Leser*innen keine nachvollziehbare lineare Narration oder Argumentation des Textes auszumachen ist ${ }^{65}$. Der Text springt sozusagen von Sequenz zu Sequenz, ist geprägt durch stete Anfänge und Abbrüche. Diese beschriebene Bewegtheit der Sprache, nach Kling das „,Sprunghafte“ (lies: Tanzende) wie das Aussparende des Gedichtes"66, wird in seinem Zweiten Daguerreotyp sowohl performativ im Text inszeniert, der auf syntaktischer und semantischer Ebene immer wieder ansetzt und abbricht, als auch diskursiv benannt als das ,fliegende, wieder und wieder anfliegende, abdrehende" (V. 22) der Schrift. Auf die Rezipierenden wirken die Sprunghaftigkeit des Textes und die abrupten Richtungswechsel der Denkbewegung mitunter wespenhaft unbe-

60. - F. von Ammon/P. Trilcke, „Einleitung“, a.a.O., S. $13 \mathrm{f}$.

61. - Vgl. zu Klings Konzept des hermetischen Schreibens P. Trilcke, Historisches Rauschen, a.a.O., S. 256-262.

62. - Wobei das Lexem ,wespenfrequenzen“" gleichzeitig auch auf eine weitere Form der rhythmisierten Anordnung des sprachlichen Materials anspielt.

63. - Ebd., S. 385.

64. - Vgl. ebd., S. 384.

65. - Vgl. ebd., S. $384 \mathrm{f}$.

66. - Thomas Kling, „Oswald von Wolkenstein“, in: Ders., Botenstoffe, Köln, DuMont, 2001, S. 147-149, hier: S. 148. 
rechenbar und verstörend. Die Lektüre kann infolgedessen einem Hin und Her zwischen der Erzeugung kurzer Evokationen ${ }^{67}$, bzw. kleinerer Bedeutungssequenzen, und deren Zerstörung bzw. Abbruch gleichen.

Durch diese Form der Sequenzierung entsteht zusätzlich ein ganz eigener Sprachrhythmus, über den Kling sagt: „Das Ganze ist natürlich vorsprachlich - ein einziges Winseln und Murren und wurmhaftes insektenhaftes Gewimmel von Lauten“"68. Trilcke hat dies die „lautbewegende Komponente der produktionsästhetischen Bewegung "69 genannt, die in ein „rhythmisches und körperlich-emotionales Erlebnis“70 des (idealen) Lesers oder der Leserin münden kann.

So verkörpern die Wespen mit ihren Bewegungen das RhythmischDisharmonische, das Stachelig-Tanzende der Dichtung Klings, die von den Lesenden sowohl ein vorsprachliches, leibliches Verstehen als auch ein intensives intellektuell-nachvollziehendes Verständnis einfordern ${ }^{71}$. Der bewegten Schrift, für welche die Wespe in Klings Werk symbolisch steht, kommen dabei die Attribute zu, die für den Dichter auch die gesprochene Sprache auszeichnen: „eine freie, gefährliche, unberechenbare, tendenziell alogische und, wie Kling immer wieder sagt: bewegliche Form der Sprachverwendung "72. Eine Konsequenz dieser spezifischen Bewegtheit ist die strukturelle Mehrdeutigkeit von Klings Lyrik, die das Gedicht für die Mitwirkung der Lesenden öffnet. Diese müssen die fragmentarischen, tendenziell alogischen Schriftsequenzen letztlich in der ,,rekonstruierende[n] Lektüre“73, zu einem sinnvollen Ganzen oder zumindest in sinnvolle Teilsequenzen zusammenfügen ${ }^{74}$. Diese Technik des teilweise unverbundenen Nebeneinanders von Sinneseindrücken ist dabei nicht als die alltägliche Wahrnehmung verfremdend, sondern als sie mit künstlerischen Mitteln imitierend zu verstehen ${ }^{75}$. Denn der insektenhafte Facettenblick und die wespenhafte Bewegtheit der Kling'schen Lyrik sollen als „Wahrnehmungsinstrument[e]“76 eine genaue Wahrnehmung von Sprache und vermittelt über diese eine genaue Wahrnehmung der - strukturell inhomogenen - Wirklichkeit

67. - Thomas Kling schreibt "Gedicht ist immer Evokation“. T. Kling, „Sprachinstallation 2“, a.a.O., S. 20.

68. - H. J. Balmes, „Lippenlesen, Ohrenbelichtung“, a.a.O., S. 20.

69. - P. Trilcke, Historisches Rauschen, a.a.O., S. 367 f., S. 374.

70. - Ebd., S. $368 \mathrm{f}$.

71. - Vgl. dazu ebd., S. 371-376.

72. - Ebd., S. 355 f.

73. - Ebd., S. 371.

74. - Möglicherweise auch unter Zuhilfenahme von Vorwissen oder durch weitere, ergänzende Recherchen, was den kurzzeitigen Ausstieg aus dem Gedichttext bedeutet, auch hier findet also ein „Anfliegen“ und „Abdrehen“ statt. Vgl. dazu ebd., S. $371 \mathrm{ff}$.

75. - Vgl. ebd., S. 388.

76. - Thomas Kling, „Stadtpläne, Stadtschriften“, in: Ders., Botenstoffe, a.a.O., S. 140-146, hier: S. 143. 
ermöglichen ${ }^{77}$. Dafür bedarf es sowohl auf Produktions- als auch auf Rezeptionsseite guter „fühler“(V. 11).

\section{Klings Schreiben als große Bejahung des Todes}

Folgt man der Fährte der Wespe als Symbol für Schöpfung und Abbruch in Klings Werk weiter, ergibt sich eine weitere, existenzielle Bedeutungsdimension der schwarz-gelben Hautflüglerin. Von Hans Jürgen Balmes nach der Wespe mit ihrem ,zeichenweiße[n] spiel“"78 in seinem Langgedicht zum Ersten Weltkrieg gefragt ${ }^{79}$, in dem diese an prominenter Stelle erscheint, antwortet Kling:

Es ist doch beeindruckend, wenn man mal sieht, wie ein Volk von Mauerwespen an ihrem Bau arbeitet, der von einem Kind abgerissen wird, ein zweiwöchiges Werk und eine Stunde später sind die ersten drei Waben schon wieder da. Das sind so Relativierungen, die mir sehr, sehr wichtig sind. Dieser Blick auf Tod, Leben, Wiederbeginn, steht niemals mit einer Coolness da, dann wäre es vollkommen unmöglich, solche Gedichte zu schreiben. Das kann ja letztlich nur aus einer Bejahung, und wenn es auch die des Todes ist, geschehen. Aus einer großen Bejahung 80 .

Klings Aussage, die zunächst recht rätselhaft anmutet, plausibilisiert den eingangs zitierten Hinweis auf die Verbindung seiner emblematisch verstandenen Wespen mit dem 16. und dem 17. Jahrhundert, welche als Jahrhunderte der vanitas gelten ${ }^{81}$. Denn sein formalästhetisches poetologisches Konzept von Sinnerzeugung und -zerstörung erhält durch den Verweis auf die unermüdliche Tätigkeit der Wespen eine zusätzliche existenzielle Dimension: die Wespen, stehen für Kling in ihrem neutralen „Blick auf Tod, Leben, Wiederbeginn“ für eine idealisierte nüchterne, geradezu stoisch-akzeptierende Haltung den existenziellen Herausforderungen des Werdens und Vergehens gegenüber. Sie sind nicht etwa, wie Kling bei Andreas Gryphius bemängelt, „tränenblind“, was „eine Durcharbeitung des Themas [verhindert]"82. Ein Vorwurf, den er wie eingangs formuliert auch

77. — Vgl. ebd., S. $142 \mathrm{f}$.

78. - T. Kling, fernhandel, a.a.O., S. 26.

79. - Vgl. ebd., S. 13-31.

80. - H. J. Balmes, „Lippenlesen, Ohrenbelichtung“, a.a.O., S. 19.

81. - Vgl. hierzu exemplarisch Ferdinand van Ingen, Vanitas und Memento mori in der deutschen Barocklyrik, Groningen, Wolters, 1966. Zur Barockrezeption bei Kling vgl. Stefanie Stockhorst, ,"Geiles 17. Jahrhundert”. Zur Barock-Rezeption Thomas Klings“, in: F. v. Ammon/P. Trilcke/A. Scharfschwert (Hrsg.), Das Gellen der Tinte..., a.a.O., S. 164-196.

82. - Thomas Kling, ,Spracharbeit, Botenstoffe. Berliner Vortrag über das 17. Jahrhundert", in: Ders., Botenstoffe, a.a.O., S. 51-69, hier: S. 54. 
gegenüber der deutschsprachigen Lyrik der 1970er Jahre erhebt, von der er sich ebenso nachdrücklich distanziert. Denn, so urteilt Kling, „[d]er Künstler [...] darf nicht vor Entsetzen starr werden, bekanntlich schadet er damit seinem Werk"83. Ähnlich wie Wagner weist Kling also die Gattungstradition der von ihm als sentimental empfundenen Erlebnislyrik weit von sich und findet in der beharrlichen Wespe das dieser Haltung entsprechende poetologische Symbol.

Den idiosynkratischen Zusammenhang zwischen formalästhetischer und existenzieller Bedeutung der Rede von Schöpfung und Abbruch, Werden und Vergehen expliziert Thomas Kling zusätzlich in seiner Laudatio für Friederike Mayröcker, die wesentlichen Einfluss auf sein eigenes Schaffen hatte, bei der Verleihung des Büchner-Preises 2001 wie folgt:

Ihre proteushaften Texte folgen einer Jean Paul verwandten Ästhetik der Abschweifung, einer Ästhetik der Unvorhersagbarkeit des Würfelwurfs, des aleatorischen Springens, die durch eine ausgefeilte Schnitttechnik erzielt wird. Sie lassen sich lesen als unmittelbare und paradoxe, dem Barock, der sprachüberschäumenden spanischen Ausformung des Barock [...] nahe Protokolle des in jedem Moment gefährdeten Glanzes der Welt; als Kommentar zu Schöpfung und Abbruch ${ }^{84}$.

Das ästhetische Verfahren der wespenhaft-unberechenbaren Bewegung, des ,aleatorischen Springens“, dessen sich Kling ebenso bedient wie Mayröcker, kann vor diesem Hintergrund ebenso als „Kommentar zu Schöpfung und Abbruch“, zu Unberechenbarkeit und Wandel der Welt gelesen werden, wie sie im Zeitalter der vanitas beharrlich beklagt wurde.

Im Bild der Wespe kulminieren diese unterschiedlichen Aspekte des Kling'schen Schreibens: Seine Ästhetik der beweglichen Sprachverwendung mit ihren unberechenbaren Wendungen verkörpern sie ebenso wie dichterische Beharrlichkeit und einen betont unsentimentalen Blick auf die Wirklichkeit, auf Schöpfungen und Abbrüche. Dass Kling als sein zentrales poetologisches Symbol nicht die traditionsbesetzte Götterbotin Honigbiene erwählte, wie es bei Jan Wagner der Fall ist, sondern ihre zorniger auftretende und daher kulturgeschichtlich unpopulärere Schwester, die Wespe, erscheint vor diesem Hintergrund konsequent. Mit jener hat er ein Symbol für seine Inszenierung von Eigensinn und Außenseitertum geschaffen, mit dem er sich und sein hermetisches dichterisches Werk sowie seine literarischen Vorbilder in einem Akt zwischen Einschreibung und Abgrenzung zugleich in einem großen literaturhistorischen Zusammenhang positioniert.

83. - Ebd.

84. - Thomas Kling, „Das euphorische Auge. Friederike Mayröcker. Auszüge aus der Laudatio zum Büchnerpreis“, in: Ders., Das brennende Archiv..., a.a.O., S. 124-125, hier: S. 124. 